\title{
Design of a superconducting rotating gantry for heavy-ion therapy
}

\author{
Y. Iwata, ${ }^{*}$ K. Noda, T. Shirai, T. Murakami, T. Furukawa, S. Mori, T. Fujita, A. Itano, \\ K. Shouda, and K. Mizushima \\ Department of Accelerator and Medical Physics, National Institute of Radiological Sciences (NIRS), \\ 4-9-1 Anagawa, Inage, Chiba 263-8555, Japan \\ T. Fujimoto \\ Accelerator Engineering Corporation (AEC), 3-8-5 Konakadai, Inage, Chiba 263-0043, Japan \\ T. Ogitsu \\ High Energy Accelerator Research Organization (KEK), 1-1 Oho, Tsukuba, Ibaraki 305-0801, Japan \\ T. Obana \\ National Institute for Fusion Science (NIFS), 322-6 Oroshi-cho, Toki-city, Gifu 509-5292, Japan \\ N. Amemiya \\ Faculty of Engineering, Kyoto University, Kyoto daigaku-Katsura, Nishikyo-ku, Kyoto 615-8530, Japan \\ T. Orikasa, S. Takami, S. Takayama, and I. Watanabe \\ Toshiba Corporation, 1-1-1 Shibaura, Minato-ku, Tokyo 105-8001, Japan \\ (Received 1 February 2012; published 10 April 2012)
}

\begin{abstract}
A superconducting rotating gantry for heavy-ion therapy is being designed. This isocentric rotating gantry can transport heavy ions with the maximum energy of $430 \mathrm{MeV} / u$ to an isocenter with irradiation angles of over 0-360 degrees, and is further capable of performing three-dimensional raster-scanning irradiation. The combined-function superconducting magnets will be employed for the rotating gantry. The superconducting magnets with optimized beam optics allow a compact gantry design with a large scan size at the isocenter; the length and the radius of the gantry will be approximately 13 and $5.5 \mathrm{~m}$, respectively, which are comparable to those for the existing proton gantries. Furthermore, the maximum scan size at the isocenter is calculated to be as large as approximately $200 \mathrm{~mm}$ square for heavy-ion beams at the maximum energy of $430 \mathrm{MeV} / u$. Based on the design of the beam optics, specifications of the superconducting magnets were determined. The superconducting magnets and magnetic-field distributions are designed using a three-dimensional field solver. With the calculated magnetic fields, beam-tracking simulations were performed to verify the design of the superconducting magnets, and concurrently to evaluate the field quality. With calculated beam profiles at the isocenter, we found that the positions of beam spots as well as their size and shape could be well reproduced as designed, proving validity of our design.
\end{abstract}

DOI: 10.1103/PhysRevSTAB.15.044701

PACS numbers: 87.56.bd, 84.71.Ba

\section{INTRODUCTION}

With the development of accelerator physics and technology, accelerators have grown to become the largest research tools over various research fields. For medical applications, large numbers of compact linear accelerators have been constructed around the world, and x rays, generated from such accelerators, have been utilized for cancer treatments. At National Institute of Radiological Sciences

\footnotetext{
*Electric address: y_iwata@nirs.go.jp (Y. Iwata)
}

Published by the American Physical Society under the terms of the Creative Commons Attribution 3.0 License. Further distribution of this work must maintain attribution to the author(s) and the published article's title, journal citation, and DOI.
(NIRS), cancer treatments using energetic carbon beams, provided from the Heavy-Ion Medical Accelerator in Chiba (HIMAC), have been performed for over 17 years, and cancer treatments of more than 6000 patients have been performed [1]. The successful results of cancer treatments have led us to construct a new treatment facility $[2,3]$. This facility is equipped with three treatment rooms; two of them have both horizontal and vertical fixedirradiation ports, and the other is a rotating-gantry port. For all ports, three-dimensional raster-scanning irradiation with a pencil beam will be employed [4-6].

In ion radiotherapy, a rotating gantry is an attractive tool, because treatment beams can be directed to a target from any of the medically desirable directions, while a patient is kept in the best position. This flexibility of beam delivery 
for this type of gantry, an isocentric rotating gantry, is advantageous to treat tumors having a wide range of tumor sites and sizes, and hence the requirements of having such a rotating gantry should increase for hospital-based therapy complexes, which will be constructed in the near future.

For proton cancer therapy, several rotating gantries were constructed around the world, and are in operation, such as the Paul Scherrer Institute (PSI) in Switzerland [7], the Loma Linda University in the U.S. [8], and three medical institutes in Japan; they are at Hyogo, Shizuoka, and Tsukuba. However, it would be very difficult to construct a rotating gantry for heavy-ion therapy, because the magnetic rigidity of carbon beams having an energy of $430 \mathrm{MeV} / u$, as required for therapy, is roughly three times higher than that for proton beams having an energy of $250 \mathrm{MeV} / u$, and therefore the size and weight for the gantry structure, including magnets and its counterweight, would become considerably larger. There is only one heavy-ion gantry, which was constructed at HIT in Heidelberg [9]; and the total weight and radius are reported to be 600 tons and $7.0 \mathrm{~m}$, respectively, while the typical weight ranges between 100 and 200 tons, and the radius is approximately $5.0 \mathrm{~m}$ for the existing proton gantries.

The aim of this study is to design a compact isocentric rotating gantry for our new treatment facility. This rotating gantry can deliver carbon ions having $430 \mathrm{MeV} / u$ to the isocenter over 360 degrees, having the capability of performing three-dimensional raster-scanning irradiation. A design key is to use combined-function superconducting magnets, thus allowing us to design a compact rotating gantry. Having optimized the layout of the gantry as well as the beam optics, the length and radius of the gantry should become approximately 13 and $5.5 \mathrm{~m}$, respectively, which are comparable to those for proton gantries. In this paper, we present the design of our rotating gantry, including the layout and the beam optics as well as the magnet design.

\section{LAYOUT AND BEAM OPTICS}

A three-dimensional image of the isocentric rotating gantry for the new treatment facility is presented in Fig. 1. This rotating gantry has a cylindrical structure with two large rings at both ends. The end rings support the total weight of the entire structure, and are placed on turning rollers so as to rotate the beam line on the rotating gantry along the central axis over 0-360 degrees. Carbon beams, provided by the HIMAC, are transported with ten sector-bending magnets, mounted on the gantry structure through each of their supporting structures; they are directed on a target located at the isocenter. In the treatment room, a tumor in a patient is precisely positioned to the isocenter by using a robotic couch.

Figure 2 shows a schematic drawing of the beam line, installed in the rotating part of the gantry. The beam line consists of ten sector-bending magnets (BM1-10), a pair of scanning magnets (SCM-X and SCM-Y), and three pairs of

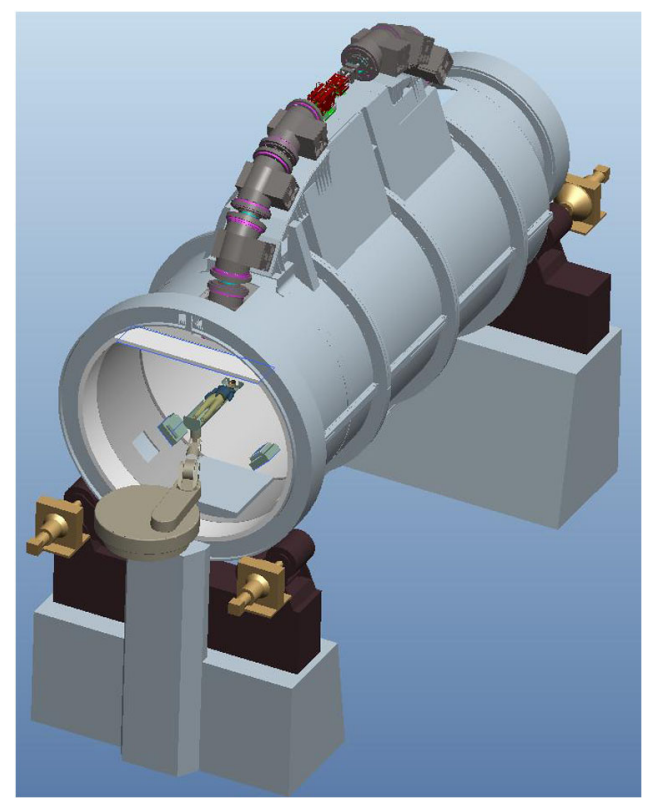

FIG. 1. Three-dimensional image of the superconducting rotating gantry for heavy-ion therapy.

steering magnets as well as a beam profile monitor (STR1-3 and PRN1-3). To design the compact gantry, combined-function superconducting magnets are to be employed for BM1-10. These superconducting magnets have a surface-winding coil structure, and can provide both dipole and quadrupole fields. The implementation of the quadrupole coil in the bending magnets makes the beam focusing without need for quadrupole magnets, enabling us to design the compact rotating gantry. The dipole and quadrupole coils are electrically isolated in the magnet, and connected to independent power supplies, so that each field component can be independently excited. Details of the magnet design are described in the following sections.

The bending radius for the first six bending magnets, BM1-6, was designed to be $\rho=2.3 \mathrm{~m}$, and corresponding magnetic-flux density to sweep a carbon beam having an energy of $430 \mathrm{MeV} / u$ is $B_{\max }=2.88 \mathrm{~T}$. To reduce the length of the rotating gantry, a total bending angle of BM1-3 or BM4-6 was determined to be $\theta=70$ degrees. However, the maximum field gradient as well as the lengths of the quadrupole field for BM1-6 were determined by considering the beam optics. To equalize the maximum field gradient over the six magnets, the lengths of the quadrupole coils, namely, the bending angles of the magnets, were optimized to be $\theta=18$ or 26 degrees, as shown in Fig. 2. Here, the quadrupole fields for each of BM1-3 and BM4-6 act to provide a D-F-D focusing cell. The maximum field gradient for this layout was found to be $G_{\max }=9.3 \mathrm{~T} / \mathrm{m}$.

Downstream of the scanning magnets, the four sectorbending magnets, BM7-10, are to be consecutively aligned. Since scanned beams traverse BM7-10, the beam envelopes had to be taken into account to determine 


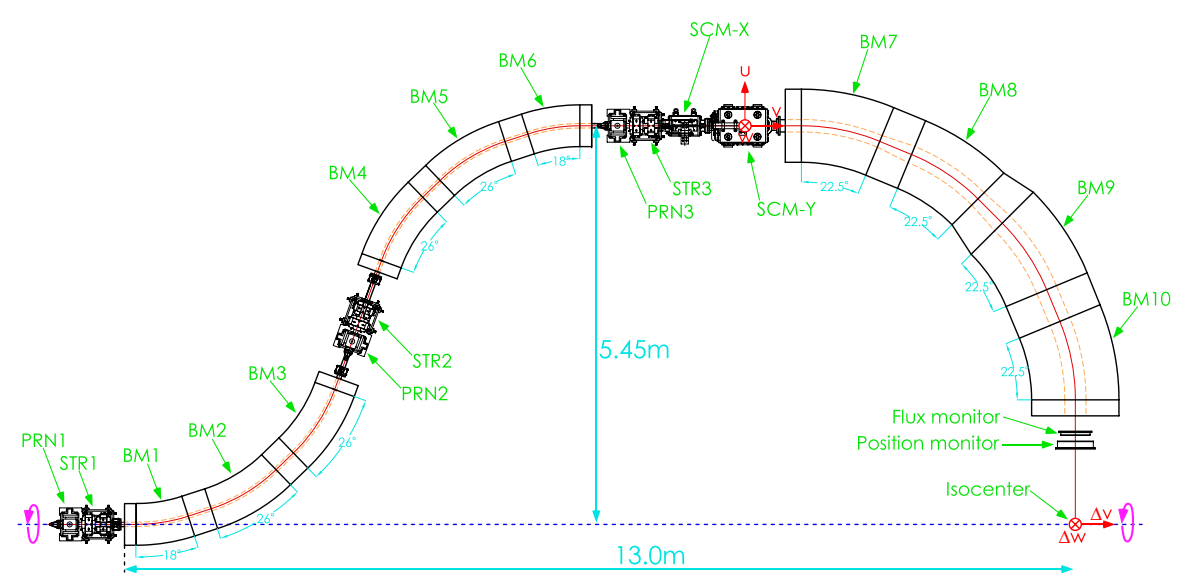

FIG. 2. Layout of the superconducting rotating gantry. The gantry consists of ten superconducting magnets (BM1-10), a pair of the scanning magnets (SCM-X and SCM-Y), and three pairs of beam profile-monitor and steering magnets (STR1-3 and PRN1-3).

the bending radius and angle of BM7-10. Since a small value of the bending radius is taken, the radius and length of the rotating gantry would be reduced; however, the maximum scan size at the isocenter may also be reduced, owing to a shorter flight path of scanned beams between the scanning magnets and the isocenter. Furthermore, scanned beams will have strong radial focusing from the bending magnets, providing a rectangular irradiation field at the isocenter. As a compromise between the sizes of the irradiation field and the gantry structure, we choose a bending radius of $2.8 \mathrm{~m}$ for BM7-10. The corresponding magnetic-flux density for a beam energy of $430 \mathrm{MeV} / u$ is 2.37 T. However, the bending angles of BM7-10 were designed to be 22.5 degrees, which was determined mostly based on manufacturing reasons. To obtain a square irradiation field at the isocenter, quadrupole coils are to be installed for BM9 and BM10. Having optimized the field gradients of BM9 and BM10, we could obtain a square irradiation field as well as parallel scanned beams at the isocenter.

Upstream of BM7, a pair of the scanning magnets, SCM-X and SCM-Y, are to be installed. The SCM-X and SCM-Y are conventional room-temperature magnets, and their basic design is identical to that described in Ref. [6]. Both SCM-X and SCM-Y are capable of sweeping beams with scanning velocities of 100 and $50 \mathrm{~mm} / \mathrm{ms}$ up to maximum deflection angles of \pm 18 and $\pm 21 \mathrm{mrad}$, respectively, for a beam energy of $430 \mathrm{MeV} / u$.

The beta and dispersion functions for the gantry beam line, presented in Fig. 2, were calculated as shown in Figs. 3(a) and 3(b), respectively. The blue and red curves in Fig. 3 represent those for the horizontal and vertical coordinates, respectively. To determine the field gradients of the quadrupole fields for BM1-6, the following matching conditions of the twiss parameters at the profile monitor, PRN1, and the isocenter were used: (i) PRN1: $\beta_{H}=\beta_{V}=$ $13 \mathrm{~m}, \quad \alpha_{H}=\alpha_{V}=0, \quad D_{H}=D_{V}=0 \mathrm{~m}, \quad$ and $D_{H}^{\prime}=$ $D^{\prime}{ }_{V}=0$; (ii) isocenter: $\beta_{H}=\beta_{V}=5 \mathrm{~m}, \alpha_{H}=\alpha_{V}=0$, $D_{H}=D_{V}=0 \mathrm{~m}$, and $D_{H}^{\prime}=D_{V}^{\prime}=0$.
However, the values of the field gradient for BM9 and BM10 were determined by the following scheme. As discussed previously, the scanned beam would have radial focusing in the horizontal coordinate, as the beam traverses BM7 through BM10, whereas there would be no focusing force in the vertical coordinate. Hence, the irradiation field would have a rectangular shape at the isocenter. To overcome radial focusing of BM7-10, the quadrupole field of BM9 and BM10 is to be utilized. The field gradients for BM9 and BM10 were optimized, so as to maximize a scan size as well as to provide parallel scanned beams at the isocenter. As a result of this optimization, we could obtain normalized field gradients for BM9 and BM10 to be $K=-0.198$ and $0.001501 / \mathrm{m}^{2}$, respectively, and were fixed in the above matching calculations.

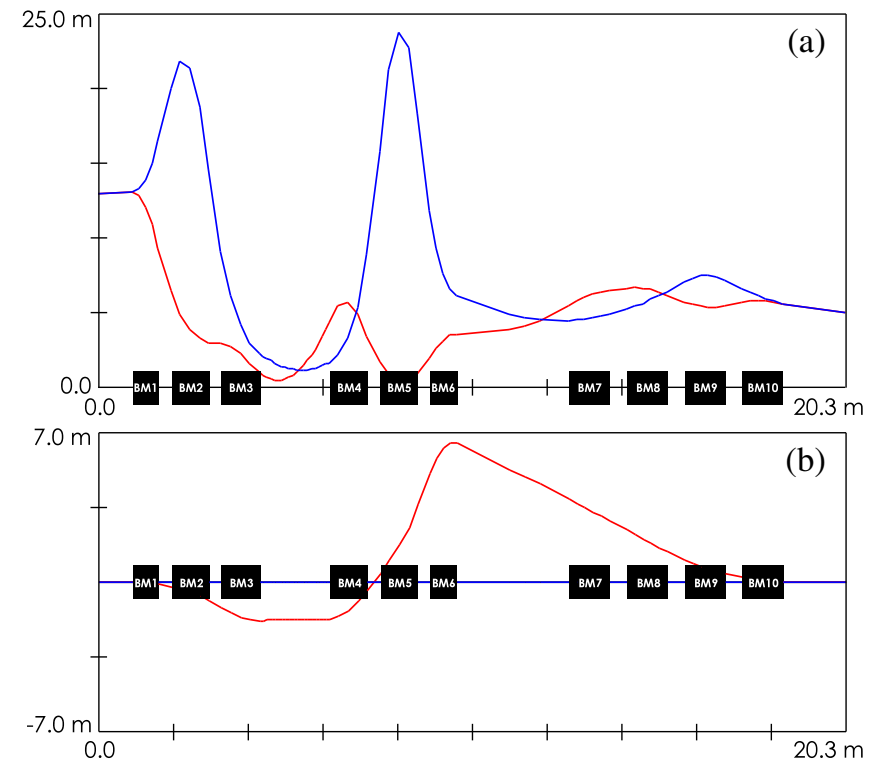

FIG. 3. (a) Beta and (b) dispersion functions of the beam line in the rotating gantry. The blue and red lines show those for the horizontal and vertical coordinates, respectively. 
TABLE I. Normalized field gradients of quadrupole magnetic field in the superconducting magnets for various conditions of the final beta, $\beta_{H, V}$, at the isocenter, as determined by opticsmatching calculations. Negative numbers denote the focusing cell.

\begin{tabular}{lcccccc}
\hline \hline & \multicolumn{5}{c}{$\mathrm{K}\left(1 / \mathrm{m}^{2}\right)$} \\
Device & $\beta_{H, V}=2 \mathrm{~m}$ & $5 \mathrm{~m}$ & $10 \mathrm{~m}$ & $15 \mathrm{~m}$ & $20 \mathrm{~m}$ & $30 \mathrm{~m}$ \\
\hline BM1 & 0.149 & 0.443 & 0.346 & 0.285 & 0.216 & 0.0534 \\
BM2 & -0.597 & -0.786 & -0.509 & -0.522 & -0.513 & -0.419 \\
BM3 & 0.826 & 0.730 & 0.286 & 0.390 & 0.473 & 0.534 \\
BM4 & 1.40 & 1.16 & 1.02 & 1.04 & 1.07 & 1.11 \\
BM5 & -1.14 & -1.16 & -1.09 & -1.13 & -1.15 & -1.18 \\
BM6 & 1.06 & 1.09 & 1.18 & 1.16 & 1.14 & 1.13 \\
BM7 & $\ldots$ & $\ldots$ & $\ldots$ & $\ldots$ & $\ldots$ & $\ldots$ \\
BM8 & $\ldots$ & $\ldots$ & $\ldots$ & $\ldots$ & $\ldots$ & $\ldots$ \\
BM9 & -0.198 & -0.198 & -0.198 & -0.198 & -0.198 & -0.198 \\
BM10 & 0.00150 & 0.00150 & 0.00150 & 0.00150 & 0.00150 & 0.00150 \\
\hline \hline
\end{tabular}

In the scanning irradiation, it is required to vary the beam size at the isocenter. To fulfill this requirement, matching calculations by changing the value of the final beta at the isocenter, while keeping the other parameters to be the same, were performed. Consequently, optical matching could be successfully made for a wide range of the final beta at the isocenter; examples of the calculated field gradients for the final beta of $\beta_{H, V}=2-30 \mathrm{~m}$ are summarized in Table I.

With the twiss functions and beam emittances, shown above, beam envelope functions can be determined. Typical values of the measured horizontal and vertical beam emittances are approximately 1 and $2 \pi \mathrm{mmmrad}$, respectively, for a beam energy of $430 \mathrm{MeV} / u$. However, the horizontal and vertical emittances of beams, as extracted from the synchrotron, generally differ from each other. For scanning irradiation using the rotating gantry, this mismatch is a concern, because the horizontal and vertical beam sizes would vary depending on the gantry angle. To compensate this mismatch, a method using a thin scatterer, as introduced in Ref. [10], will be employed. This method enables us to compensate for the asymmetric

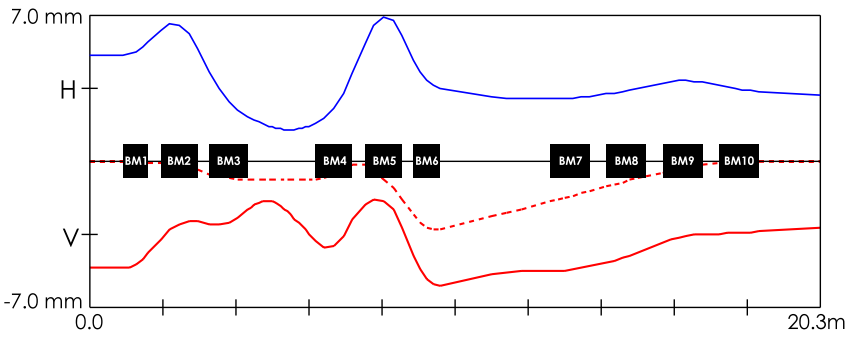

FIG. 4. Blue and red lines show the horizontal and vertical beam envelope functions, respectively. The solid and dashes lines represent betatron and momentum envelopes, respectively. In the calculations, typical emittances of $\varepsilon_{H}=\varepsilon_{V}=$ $2 \pi \mathrm{mm}$ mrad and momentum spread of $\Delta p / p= \pm 5 \times 10^{-4}$ for beams having $430 \mathrm{MeV} / u$ were used. phase-space distributions, and hence to provide symmetric beam emittances at the entrance of the rotating gantry.

Assuming equal horizontal and vertical emittances of $\varepsilon_{H}=\varepsilon_{V}=2 \pi \mathrm{mmmrad}$, and a momentum spread of $\Delta p / p= \pm 5 \times 10^{-4}$, and using the beta and dispersion functions, as given in Fig. 3, the horizontal and vertical envelope functions were calculated, as shown by the blue and red curves in Fig. 4, where the betatron and momentum envelope functions are indicated by the solid and dashed curves, respectively. We can see from the figure that the beam size is within $\pm 7.0 \mathrm{~mm}$. Considering that the beam emittances adiabatically increase as the beam energy decreases, the beam emittance for $80 \mathrm{MeV} / u$ is estimated
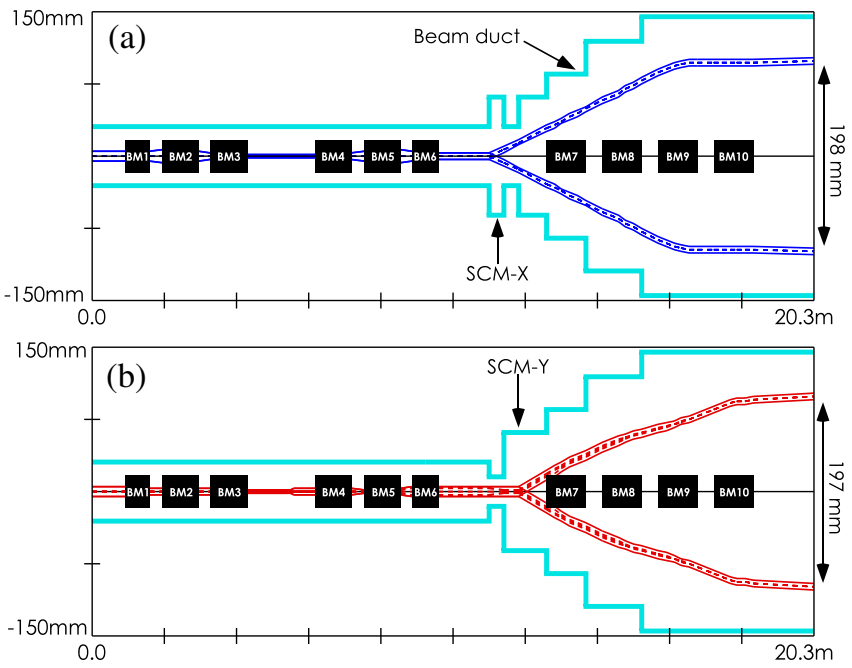

FIG. 5. (a) Horizontal and (b) vertical beam envelope functions with kicks of the scanning magnets, SCM-X and SCM-Y. The maximum kick angles of \pm 18 and $\pm 21 \mathrm{mrad}$ for beams having $430 \mathrm{MeV} / u$ were applied for SCM-X and SCM-Y, respectively. In the calculations, typical values of matched emittances, $\varepsilon_{H}=\varepsilon_{V}=2 \pi \mathrm{mm} \mathrm{mrad}$, and momentum spread, $\Delta p / p= \pm 5 \times 10^{-4}$, for beams having $430 \mathrm{MeV} / u$ were assumed. 
TABLE II. Summary of parameters for the superconducting magnets, as determined by beam-optics calculations.

\begin{tabular}{|c|c|c|c|c|c|c|c|}
\hline Parameter & Symbol & BM1 and BM6 & BM2-BM5 & BM7 & BM8 & BM9 and BM10 & Unit \\
\hline Bending angle & $\theta$ & 18 & 26 & 22.5 & 22.5 & 22.5 & deg \\
\hline Bending radius & $\rho$ & 2.3 & 2.3 & 2.8 & 2.8 & 2.8 & $\mathrm{~m}$ \\
\hline Bore radius & $R_{0}$ & 30 & 30 & 85 & 120 & 145 & $\mathrm{~mm}$ \\
\hline Reference radius or effective area & $r_{0}$ or $A_{0}$ & 20 & 20 & $60 \times 60$ & $80 \times 80$ & $100 \times 100$ & $\mathrm{~mm}$ or $\mathrm{mm}^{2}$ \\
\hline Magnetic flux density (dipole field) & $B_{\max }$ & 2.88 & 2.88 & 2.37 & 2.37 & 2.37 & $\mathrm{~m}$ \\
\hline Maximum field gradient (quadrupole field) & $G_{\max }$ & 9.0 & 9.0 & $\ldots$ & $\cdots$ & 1.3 & $\mathrm{~T} / \mathrm{m}$ \\
\hline Field uniformity (dipole field) & $|\Delta B L / B L|$ & & & $1 \times 10^{-4}$ & & & $\ldots$ \\
\hline Field uniformity (quadrupole field) & $|\Delta G L / G L|$ & & & $1 \times 10^{-3}$ & & & $\cdots$ \\
\hline
\end{tabular}

to be 2.5 times larger than that for $430 \mathrm{MeV} / u$, and the corresponding beam size for $80 \mathrm{MeV} / u$ would be evaluated to be $\pm 11 \mathrm{~mm}$.

Figure 5 shows similar plots as in Fig. 4, but with kicks of SCM-X and SCM-Y. In the calculations, the same values of the beam emittances and momentum spread were employed. However, the deflection angles of the SCM-X and SCM-Y were taken to be \pm 18 and $\pm 21 \mathrm{mrad}$, respectively. As can be seen in the figure, we could obtained a scanned beam having a maximum scan size of approximately $200 \mathrm{~mm}$ square, while keeping the defocusing beam angle to be less than $2 \mathrm{mrad}$ at the isocenter. Finally, the parameters for the magnets, as determined by the beam-optics calculations, are summarized in Table II.

\section{DESIGN AND FIELD CALCULATIONS OF SUPERCONDUCTING MAGNETS}

The superconducting magnets for the beam line on the rotating gantry are categorized into two groups based on their apertures, namely, small- and large-aperture magnets. The small-aperture magnets are BM1-6, and are located upstream of the scanning magnets. The bore radius of the small-aperture magnets is $30 \mathrm{~mm}$. However, the largeaperture magnets are BM7-10; they are located downstream of the scanning magnets. Since scanned beams would traverse BM7-10, their bore radii ranges between 85 and $145 \mathrm{~mm}$, depending of the magnet location. Subsequently, the small- and large-aperture magnets can be subdivided into five kinds, as summarized in Table II. In this section, the design as well as results of threedimensional magnetic-field calculations for both the smalland large-aperture magnets are described.

\section{A. Small-aperture magnet}

The superconducting magnets of BM1-6 have a bore radius of $30 \mathrm{~mm}$, and are used to transport the beam to the scanning magnets. Considering the beam size, as estimated by beam optics, the reference radius of BM1-6 was design to be $r_{0}=20 \mathrm{~mm}$. As mentioned previously, the coil of the magnets has a surface-winding structure, and has both dipole and quadrupole superconducting coils to provide beam sweeping as well as focusing. Since BM1-6 would have an identical structure and specifications, except for their bending radius of either 18 or 26 degrees, we will show here the design only for a 26-degree magnet, namely, BM2-5.

A cross-sectional view of the small-aperture magnets, BM1-6, is shown in Fig. 6. The diameter of the beam duct, which is to be made of stainless steel, was designed to be $\phi 60 \mathrm{~mm}$. The thermal insulators are to be wrapped around the beam duct to prevent heat penetration. Outside of the beam duct, a coil base, which is also to be made of stainless steel, is present, and superconducting NbTi wires, having a diameter of $0.9 \mathrm{~mm}$, are to be wounded on the coil base. As it can be seen in the figure, the superconducting coil has a layered structure. The positions of the superconducting wires for the first 8 layers has a $\cos (2 \theta)$ distribution, and are optimized to produce a pure quadrupole field $[11,12]$. Having glued an aluminum sheet with a thickness of $1 \mathrm{~mm}$ on the quadrupole coil, 26 layers of the superconducting dipole coil are subsequently wound over the aluminum sheet. Similarly, the positions of the superconducting wires for these dipole layers have a $\cos \theta$ distribution, and are optimized so as to produce a pure dipole field. The superconducting coil has 34 layers with 3426 turns/pole for the dipole coil and 400 turns/pole for the quadrupole coil. The dipole and quadrupole coils are electrically isolated, and hence can be independently excited.

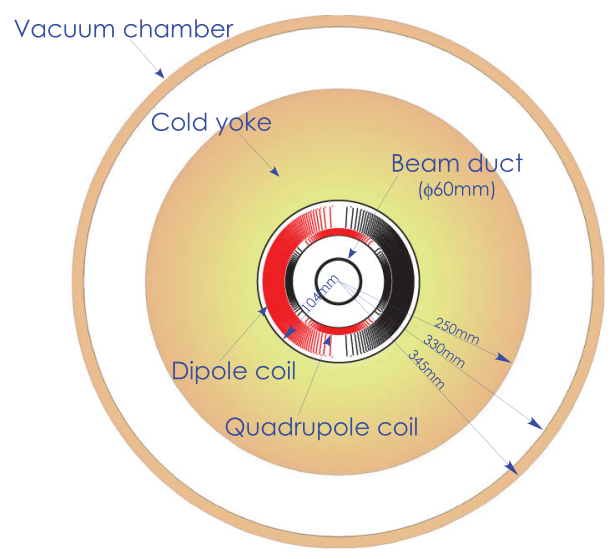

FIG. 6. Cross-sectional view of the superconducting magnets for BM1-BM6. 

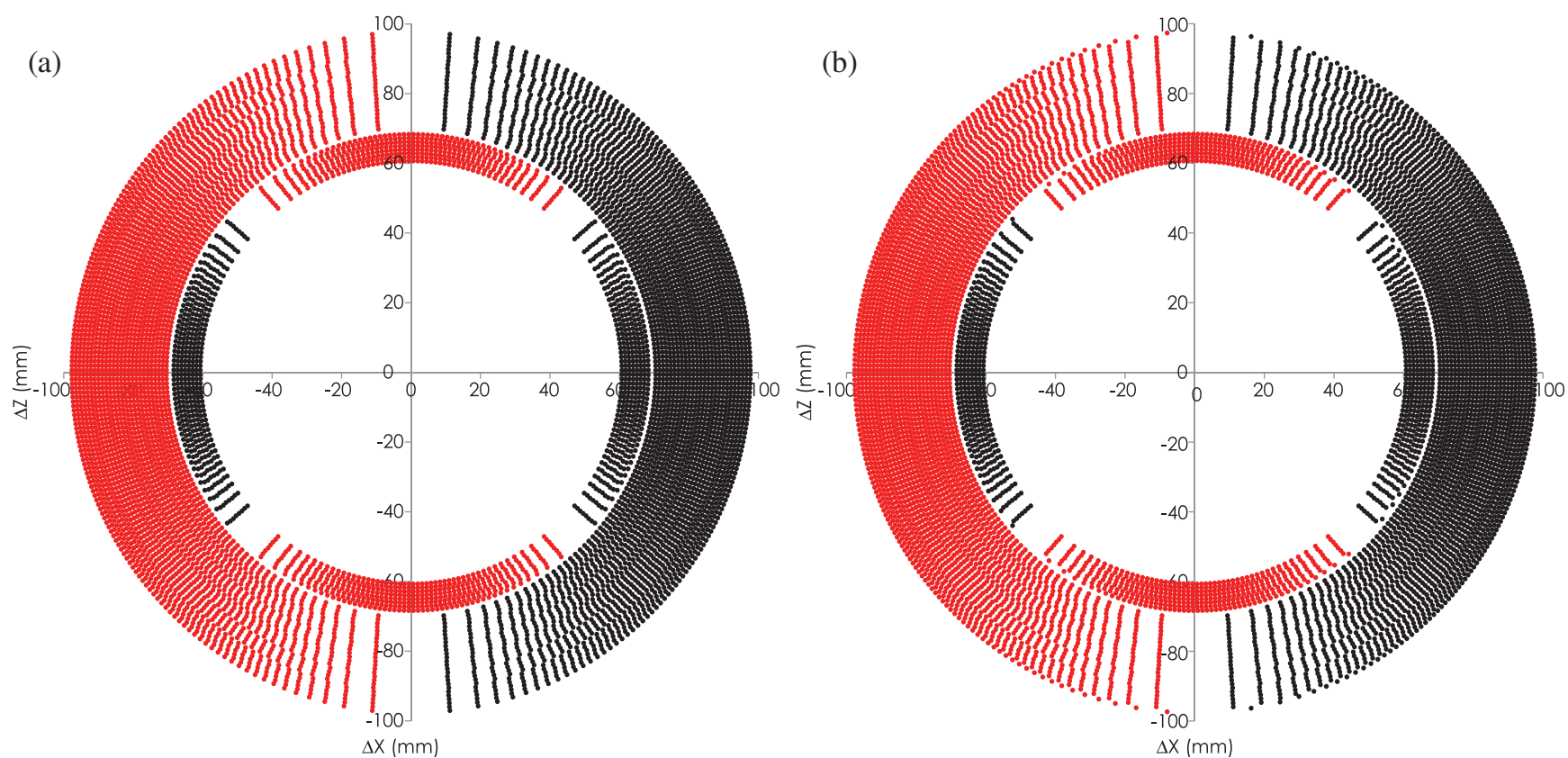

FIG. 7. Profile of the superconducting coil for the magnets of BM1-6 (a) before and (b) after a correction. The coil consisted of the 8 layers of the quadrupole coils and 26 layers of dipole coils. The dipole and quadrupole coils were electrically isolated, and can be independently excited.

The coil would be covered by a cold yoke with a thickness of $146 \mathrm{~mm}$, and are to be installed in the vacuum chamber through supports, made by glass-fiber-reinforced plastics for thermal insulation. Further, the coil and cold yoke as well as a thermal shield between them are to be kept in vacuum. The cold yoke would be made of laminated steel so as to suppress eddy currents, which may originate during fast lumping of the magnetic fields in multiple-energy operation with extended flattops, as described in Ref. [13]. The dimensions of the coil, yoke, and vacuum chamber are summarized in Table III.

Figure 7(a) shows a profile of the superconducting coils. As it was mentioned previously, the positions of superconductors in each layer were optimized in twodimensional coordinates, so as to provide pure dipole or quadrupole fields. However, to estimate the effect of the cold yoke as well as the three-dimensional structure of the magnet, we performed three-dimensional magnetic-field

TABLE III. Dimensions of the coil, yoke, and vacuum chamber for the superconducting magnets.

\begin{tabular}{lcc}
\hline \hline & \multicolumn{2}{c}{ Value $(\mathrm{mm})$} \\
Parameter & BM1-6 & BM9/10 \\
\hline Inner radius of coil & 60.7 & 175.9 \\
Outer radius of coil & 97.7 & 208.8 \\
Inner radius of yoke & 104.0 & 230.0 \\
Outer radius of yoke & 250.0 & 500.0 \\
Inner radius of vacuum chamber & 330.0 & 600.0 \\
Outer radius of vacuum chamber & 345.0 & 620.0 \\
\hline \hline
\end{tabular}

calculations using the OPERA-3D code [14]. In the code, 3426 turns/pole for the curved dipole coil and 400 turns/pole for the curved quadrupole coil as well as the cold yoke and vacuum chamber were precisely modeled, and a three-dimensional magnetic field was calculated.

A three-dimensional image of the magnetic-flux density, as calculated by the OPERA-3D code, is presented in Fig. 8. The maximum magnetic-flux density on the yoke was

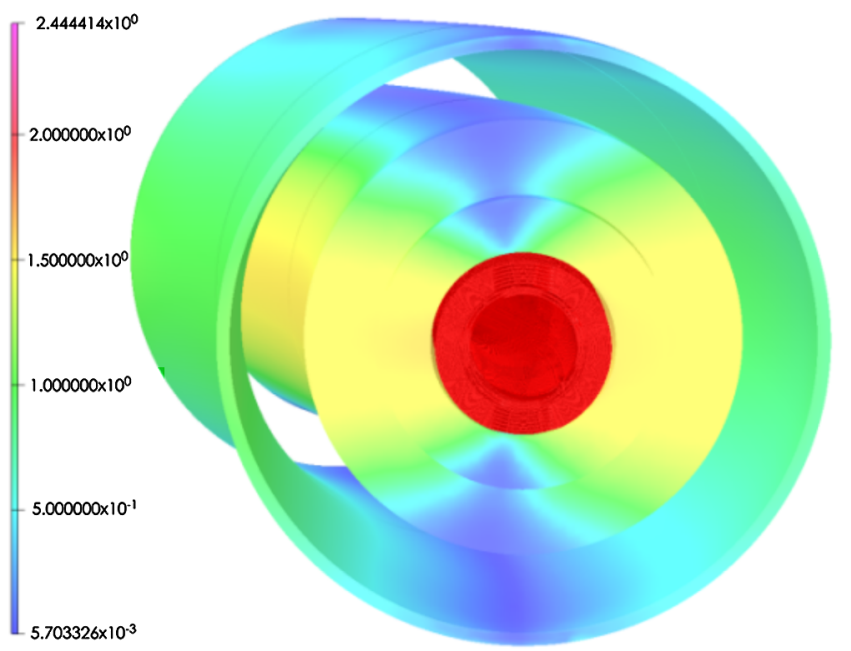

FIG. 8. Three-dimensional image of magnetic-flux density for the small-aperture magnet, as calculated by the threedimensional electromagnetic field solver, OPERA-3D. The unit of the magnetic field, $|B|$, is T. In the calculation, coil currents of 136 and 130 A were applied for the dipole and quadrupole coils, respectively. 

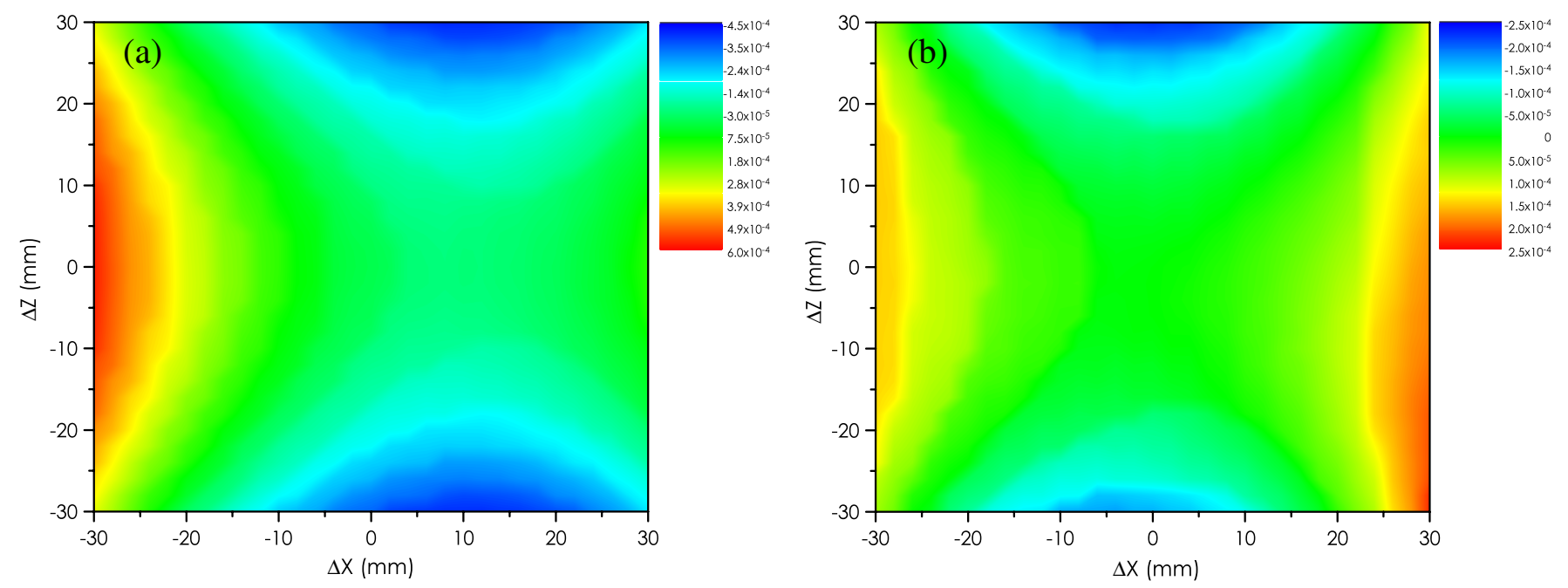

FIG. 9. Uniformity of the BL products, $\Delta B_{z} L / B_{z} L$, of the dipole field for the small-aperture magnet (a) before and (b) after corrections.

observed to be $2.44 \mathrm{~T}$. In the calculation, coil currents of 136 and 130 A were applied for the dipole and quadrupole coils, respectively. With these currents, we obtained the dipole field and field gradient, as integrated along the central beam trajectory, to be $B_{z} L=3.01 \mathrm{Tm}$ and $G L=$ $10.3 \mathrm{~T}$, respectively, which would satisfy the required specification, as given in Table II.

To evaluate the field quality of the dipole field, mapping of the vertical magnetic-flux density, as integrated along a beam trajectory, $B_{z} L$, was performed. The threedimensional magnetic field, exciting only the dipole coil, was initially calculated for a coil current of $I=136 \mathrm{~A}$. Then, the BL products, $B_{z} L$, of various beam trajectories, as shifted horizontally by $\Delta X$ and vertically by $\Delta Z$ from the center beam trajectory, were calculated, and further converted to the field uniformity, $\Delta B_{z} L / B_{z} L$. A map of the field uniformity for the dipole field, as determined by the above calculations, is shown in Fig. 9(a). From this figure, we observed that higher-order multipole fields, which are composed mostly of quadrupole and sextupole fields, exist, and that the uniformity within the reference radius of $r_{0}=$ $20 \mathrm{~mm}$ is $\left|\Delta B_{z} L / B_{z} L\right| \leq 3.2 \times 10^{-4}$, which would not satisfy the required uniformity. Since these multipole fields were not present in two-dimensional magnetic-field calculations, we would presume that the multipole fields originated from the curved structure of the magnet as well as the coil ends.

To improve the field uniformity, the conductor positions for the outermost layer of the dipole coil were modified, so that the multipole field, produced by this layer, compensates the higher-order multipole fields, as observed in Fig. 9(a). A profile of the modified coil is shown in Fig. 7(b). As can be seen in the figure, the outermost coil layer for the dipole coil has an asymmetric shape, indicating that this layer would produce a quadrupole field for the correction. With the modified coil profile, a similar field calculation using OPERA-3D was performed; the result is shown in Fig. 9(b). We can see that the field uniformity is significantly improved to be $\left|\Delta B_{z} L / B_{z} L\right| \leq 1.0 \times 10^{-4}$ within the reference radius.

A similar analysis, as done for the dipole coil, was made to design the quadrupole. The three-dimensional magnetic field, created only by the quadrupole coil, as shown in Fig. 7(a), was initially calculated. The GL products, $G L$, of various beam trajectories, as shifted horizontally by $\Delta X$ and vertically by $\Delta Z$ from the center beam trajectory, were calculated, and converted to the field uniformity, $\Delta G L / G L$. The calculated results of the horizontal and vertical field gradients, integrated along the beam trajectories, are given in Fig. 10. Here, the horizontal and vertical field gradients are defined as $G_{x} L=d\left(B_{x} L\right) / d z$ and $G_{z}=d\left(B_{z} L\right) / d x$, respectively. In the calculation, a coil current of $I=130$ A was applied, providing a field gradient of $G=9.04 \mathrm{~T} / \mathrm{m}$ in the middle of the magnet and $G L=10.4 \mathrm{~T}$ along the center beam trajectory. The observed uniformity of the field gradient was $|\Delta G L / G L| \leq$ $2.5 \times 10^{-3}$ within the reference radius, whereas a uniformity of $|\Delta G L / G L| \leq 1 \times 10^{-3}$ is needed, as prescribed in Table II.

This heterogeneous field can be corrected by the same method as performed in the design of the dipole coil. The conductor positions for the outermost layer of the quadrupole coil were corrected, so as to compensate the observed higher-order multipole fields. As a result, we obtained the coil profile after a correction, as presented in Fig. 7(b). Having used the corrected coil profile in the threedimensional field calculation, we consequently obtained the uniformity, as shown in Fig. 11. As was found in the figure, the uniformity was remarkably improved to $|\Delta G L / G L| \leq 1.2 \times 10^{-4}$. 

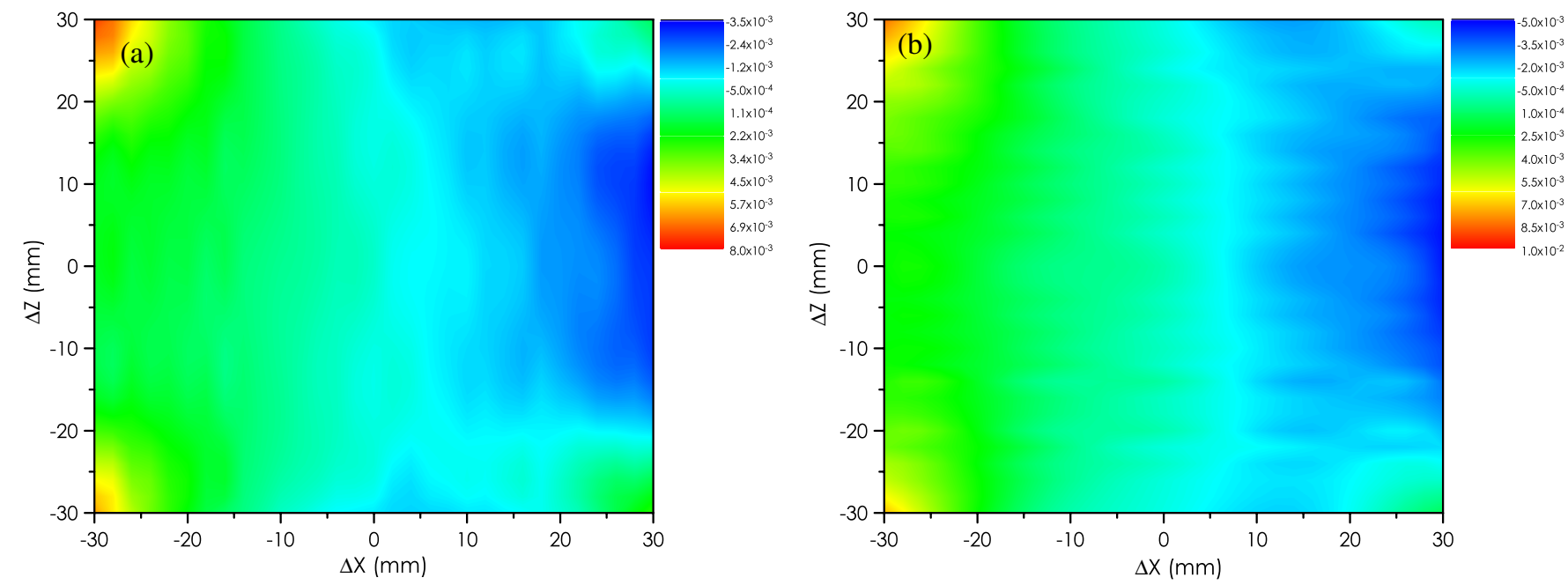

FIG. 10. Uniformity of the (a) horizontal GL, $\Delta G_{x} L / G_{x} L$, and (b) vertical GL, $\Delta G_{z} L / G_{z} L$, products along beam trajectory for the small-aperture magnet before corrections.
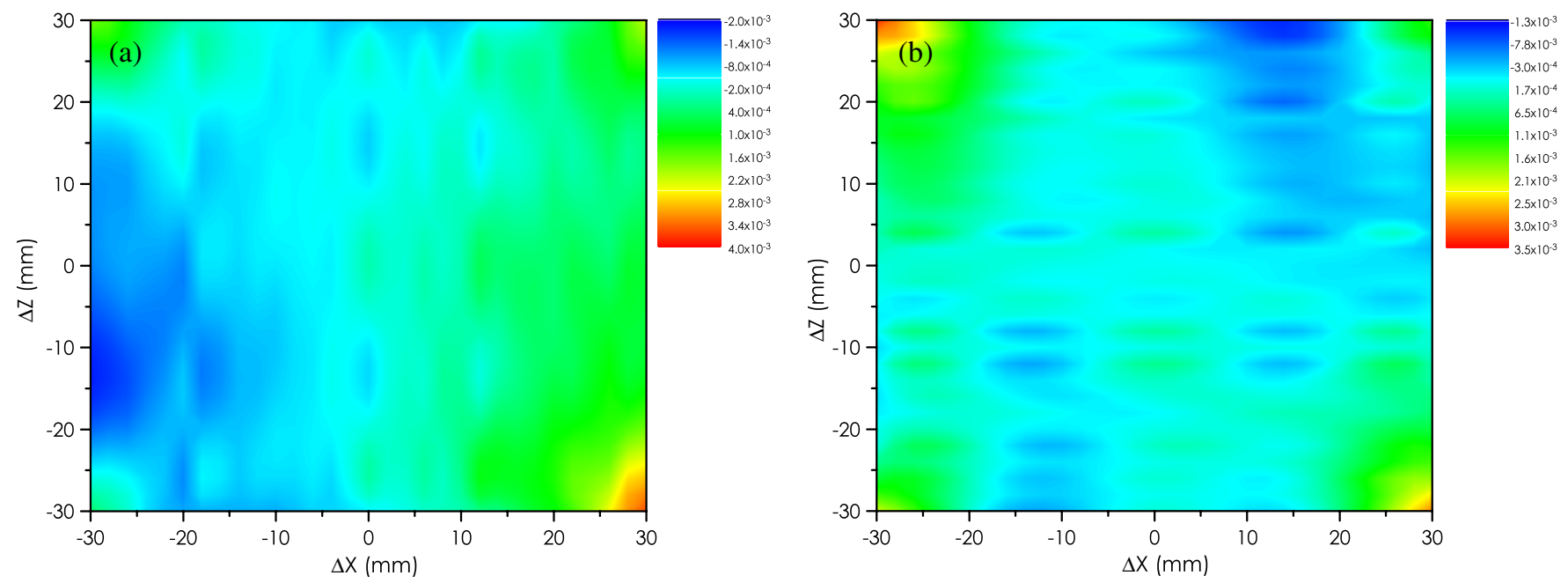

FIG. 11. Uniformity of the (a) horizontal GL, $\Delta G_{x} L / G_{x} L$, and (b) vertical GL, $\Delta G_{z} L / G_{z} L$, products along the beam trajectory, for the small-aperture magnet after corrections.

Since large numbers of conductors are to be wounded, an effect of mechanical errors in a winding process to field uniformity is a concern. To clarify this, field calculations with and without such errors were made, and an effect to field uniformity was investigated. In calculations, random errors in conductor positions to the angular direction were assumed, because mechanical errors in the angular direction would be more probable in a winding process than those in the radial direction. Results of the field calculations indicated that random errors of $\sigma=0.1 \mathrm{~mm}$ would produce an error to the field uniformity of $\leq 4 \times 10^{-5}$ within the reference radius. Considering the typical mechanical error in a winding process would not exceed $\sigma=$ $0.1 \mathrm{~mm}$, we concluded that the effect of the mechanical errors in a winding process should be negligible.
In treatments using the rotating gantry, the beam energy would be varied from 430 down to $80 \mathrm{MeV} / u$, and the corresponding currents of the dipole coil would be $I=$ $136 \mathrm{~A}$ and $54 \mathrm{~A}$, respectively. Hence, it is required to produce a homogeneous magnetic field over this current range. To clarify this, the field uniformity was calculated for the various currents for the dipole coil along the $\Delta X$ and $\Delta Z$ axes, as shown in Fig. 12. Considering that the uniformity would not change below the coil current of $I=110 \mathrm{~A}$, it can be concluded that the uniformity is better than $\left|\Delta B_{z} L / B_{z} L\right| \leq 1 \times 10^{-4}$ within the reference radius over the entire energy range. Finally, we note here that the same analysis for the quadrupole field suggested that the field gradient of the quadrupole field would not vary depending on the coil current of the quadrupole coil. 

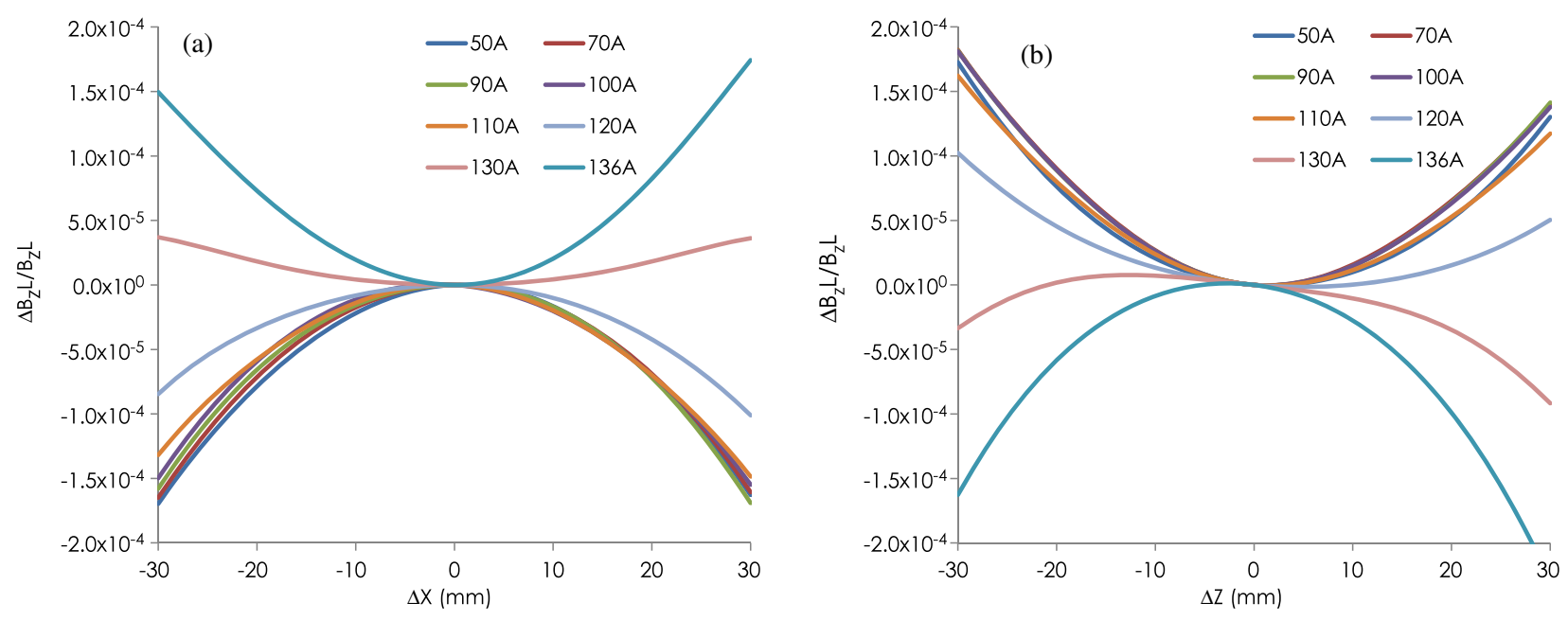

FIG. 12. Uniformity of BL products, $\Delta B_{z} L / B_{z} L$, of the dipole field for various coil currents of the dipole coil along (a) the $X$ axis and (b) the $Z$ axis, as determined by the three-dimensional field calculations.
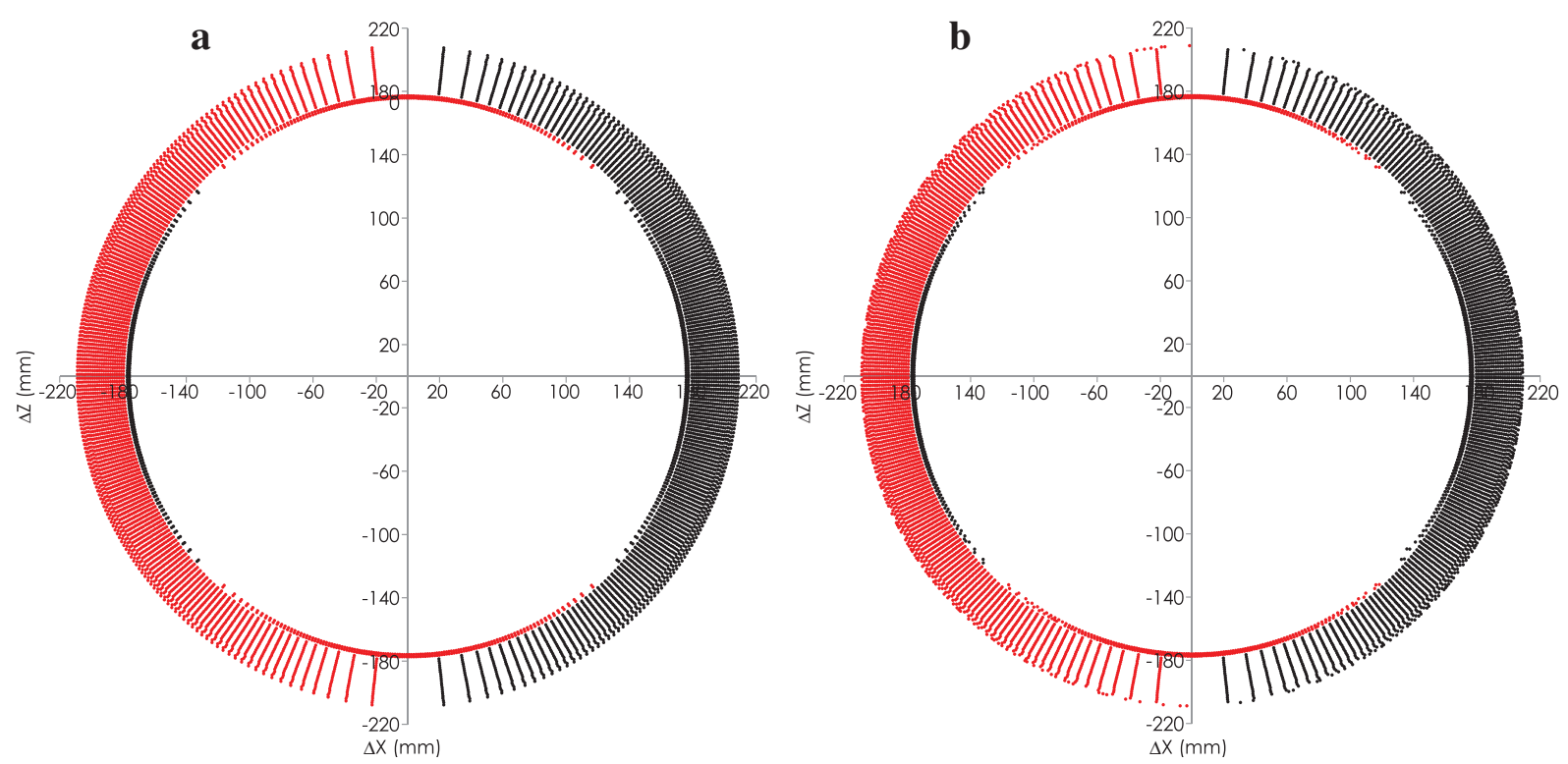

FIG. 13. Profile of the superconducting coil for the magnets of BM9 and BM10 (a) before and (b) after corrections. The coil consists of 2 layers for the quadrupole coils and 22 layers for the dipole coils. The dipole and quadrupole coils were electrically isolated, and can be independently excited.

\section{B. Large-aperture magnet}

The superconducting magnets, categorized as largeaperture magnets, are those installed downstream of the scanning magnets. Since a scanned beam traverses these magnets, the bore radius as well as the reference radius for them had to be designed so as to accept all of the scanned beams, as summarized in Table II. Since the BM9 and BM10 have the largest bore radius, we will show here the design for BM9 and BM10.

As discussed in the preceding section, BM9 and BM10 have both dipole and quadrupole coils. Considering that the maximum beam size of scanned beams, and that the irradiation field has a square shape, the dipole and quadrupole fields must be homogeneous over an effective area of $100 \mathrm{~mm} \times 100 \mathrm{~mm}$. Further, to accept scanned beams, the bore radius was design to be $145 \mathrm{~mm}$.

The cross-sectional structure of the large-aperture magnet is the same as those for the small-aperture magnet, while the dimensions of the coil, yoke, and vacuum chamber are enlarged, as summarized in Table III. Furthermore, the coil structure has the same layered structure, while it consists of 2 quadrupole layers and 22 dipole layers, as shown in Fig. 13; the $1.2 \mathrm{~mm}$ diameter superconducting $\mathrm{NbTi}$ wires are to be used for this magnet. The superconducting coil totally has 24 layers with 3702 turns/pole for the dipole coil and 240 turns/pole for the quadrupole coil. The dipole 


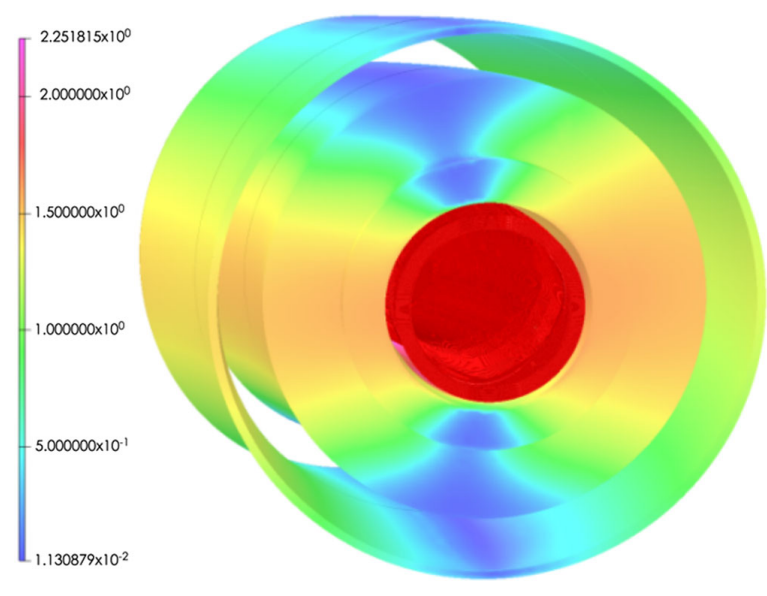

FIG. 14. Three-dimensional image of magnetic-flux density for the large-aperture magnet, as calculated by the threedimensional electromagnetic field solver, OPERA-3D. The unit of the magnetic field, $|B|$, is Tesla. In the calculation, the coil currents of 231 and 200 A were applied for the dipole and quadrupole coils, respectively.

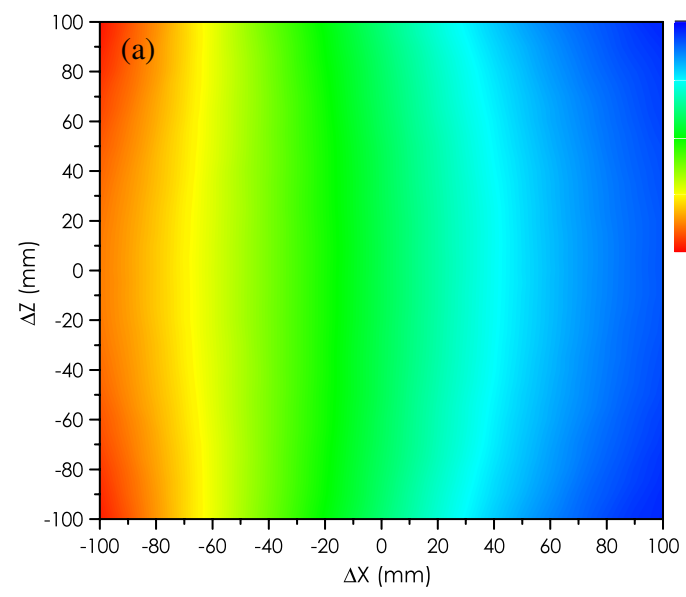

and quadrupole coils are also to be electrically isolated, and therefore can be independently excited.

Three-dimensional magnetic-field calculations, using the OPERA-3D code, were performed for this magnet. A three-dimensional model of the magnet, based on the coil profile, as presented in Fig. 13(a), was created and solved. A three-dimensional image of the magnetic-flux density, as calculated by the OPERA-3D, is presented in Fig. 14. The maximum magnetic-flux density on the yoke was observed to be $2.25 \mathrm{~T}$, where coil currents of 231 and $200 \mathrm{~A}$ were applied to the dipole and quadrupole coils, respectively. A map of the field uniformity for the dipole field, $\Delta B_{z} L / B_{z} L$, obtained with analysis of the calculation, exciting only the dipole coil, is presented in Fig. 15(a). In the calculation, a coil current of $I=231$ A was applied, providing $B_{z}=$ $2.37 \mathrm{~T}$ on the center of the magnet and $B_{z} L=2.60 \mathrm{Tm}$ for the center beam trajectory. As can be seen from the map, the calculated field has multipole fields, and that the uniformity is observed to be $\left|\Delta B_{z} L / B_{z} L\right| \leq 1.6 \times 10^{-3}$ over the effective area of $100 \mathrm{~mm} \times 100 \mathrm{~mm}$; this would

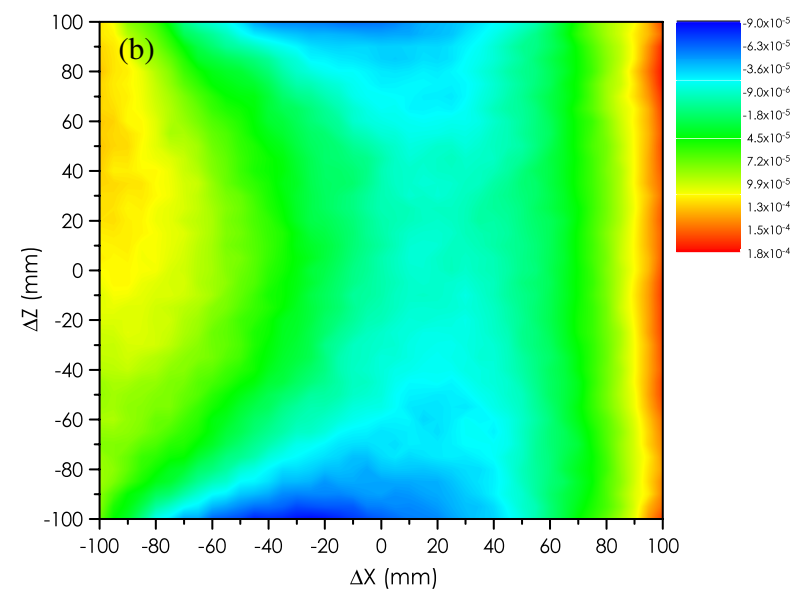

FIG. 15. Uniformity of the BL products, $\Delta B_{z} L / B_{z} L$, of the dipole field for the large-aperture magnet (a) before and (b) after corrections.
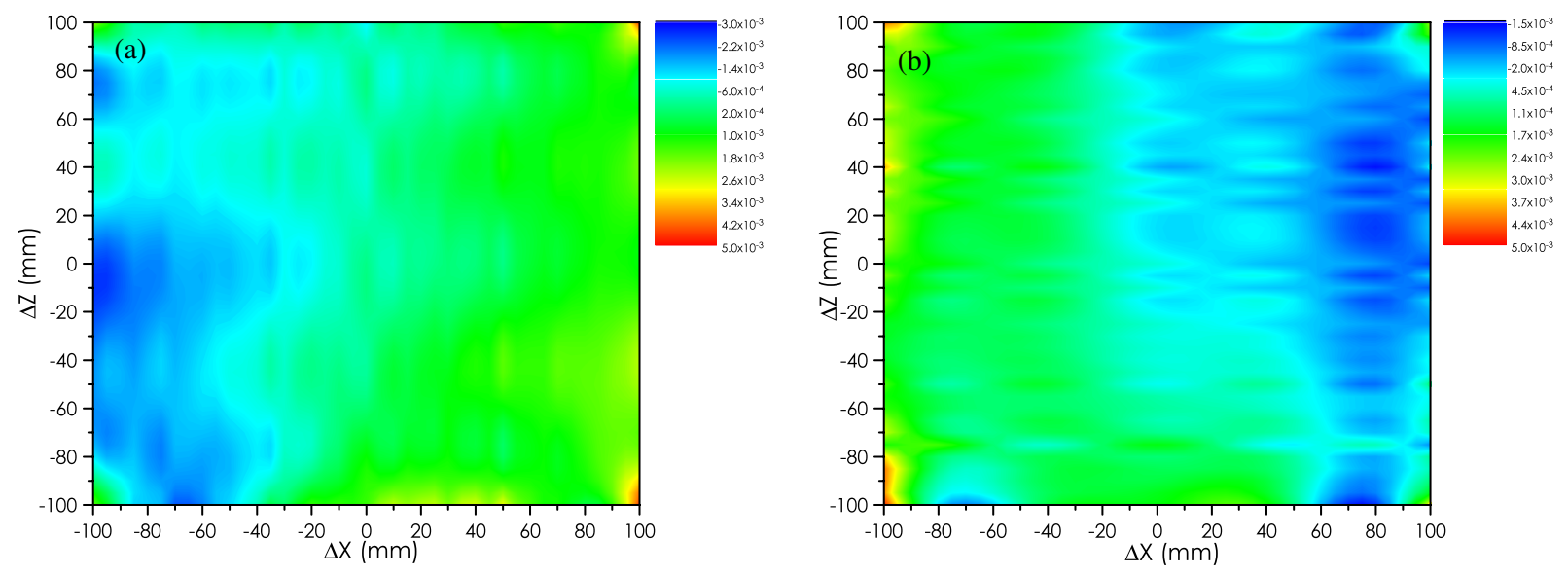

FIG. 16. Uniformity of the (a) horizontal GL, $\Delta G_{x} L / G_{x} L$, and (b) vertical GL, $\Delta G_{z} L / G_{z} L$, products along beam trajectory for the large-aperture magnet after corrections. 

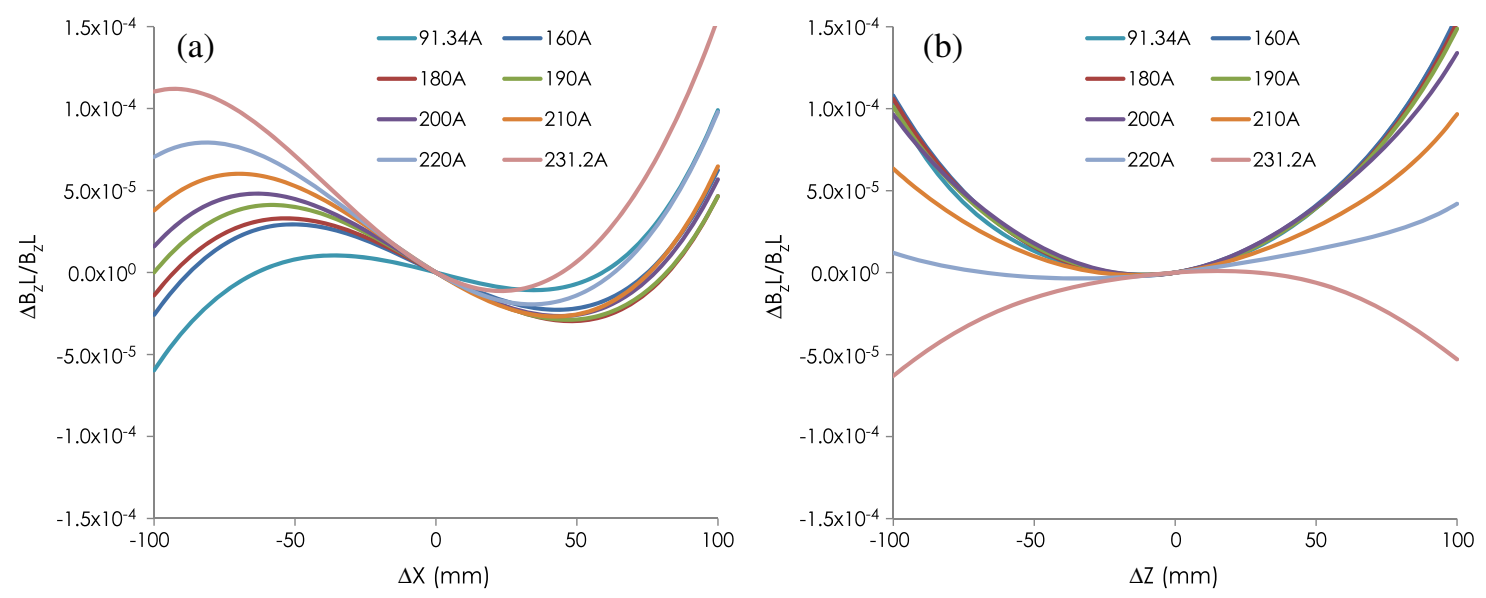

FIG. 17. Uniformity of BL products, $\Delta B_{z} L / B_{z} L$, of the dipole field for various coil currents of the dipole coil along (a) the $X$ axis and (b) the $Z$ axis, as determined by the three-dimensional field calculations.

not suffice the required uniformity, as prescribed to be $\left|\Delta B_{z} L / B_{z} L\right| \leq 1 \times 10^{-4}$.

To improve the field uniformity, we first formulated a similar method, as used for the small-aperture magnet; that is, the conductor positions for the outermost layer of the dipole coil were corrected so as to produce a multipole field, and thus to compensate for the observed higher-order multipole fields. However, the result of the calculation using the above method was not satisfactory, since we had observed appreciable field errors of $\left|\Delta B_{z} L / B_{z} L\right| \leq 6.0 \times 10^{-4}$ on the edge of the effective area. To obtain a homogeneous field over the entire effective area, we optimized the conductor positions of the outermost layer, so as to compensate the observed error in the uniformity over the entire effective area, as can be observed in Fig. 15(a). A result of this optimization provided the coil profile, as given in Fig. 13(b). Subsequently, a three-dimensional field calculation based on the optimized coil profile yielded a map of the uniformity, as provided in Fig. 15(b). In the calculation, a coil current of $I=231.2$ A was applied, providing $B_{z}=$ $2.37 \mathrm{~T}$ on the center of the magnet and $B_{z} L=2.61 \mathrm{Tm}$ for the center beam trajectory. Consequently, the uniformity is substantially improved to $\left|\Delta B_{z} L / B_{z} L\right| \leq 1.8 \times 10^{-4}$ over the entire effective area, although this does not completely fulfill the required uniformity.

The same optimization method of conductor positions had been employed for the quadrupole coil. Figures 13(a) and 13(b) show the coil profile before and after optimization. In the calculation, a coil current of $I=200$ A was applied, providing a field gradient of $G=1.30 \mathrm{~T} / \mathrm{m}$ in the middle of the magnet and $G L=1.51 \mathrm{~T}$ along the center beam trajectory. The uniformity of the field gradient, $\Delta G L / G L$, after optimization is given in Fig. 16. We obtained a homogeneous field gradient for most parts of the effective area.

Finally, calculations of the field uniformity for various coil currents were made. Since the beam energies to be used for treatments range between 80 and $430 \mathrm{MeV} / u$, the calculations were made between the corresponding cur- rents for the dipole coil; they are 91.34 and $231.2 \mathrm{~A}$, respectively. Figures 17 (a) and 17 (b) show the uniformity of the dipole field, $\Delta B_{z} L / B_{z} L$, along the $\Delta X$ and $\Delta Z$ axes, respectively. We found that the uniformity would not vary under a coil current of $I=200 \mathrm{~A}$, and that concurrently the change in the uniformity is tolerable for this current range. Further, the same analysis was made for the quadrupole field, and a result of the analysis proved that the distributions of the field gradient are stable over the coil currents. This indicates that this magnet provides a satisfactory field quality, as expected.

\section{BEAM-TRACKING SIMULATIONS}

To confirm the design of the superconducting magnets, and concurrently to evaluate the field quality, as provided by three-dimensional calculations, we performed beamtracking simulations. Since the quality of scanned beams is quite important for treatments using the scanning irradiation method, tracking simulations were made downstream of the scanning magnets; a tracking of beam particles initiated in the middle of SCM-Y, and beam trajectories were calculated through BM7-BM10, before reaching the isocenter.

Tracking simulations were made to reproduce the calculated beam optics for the matching condition, as plotted in Fig. 3. With a calculation on the beam optics, the twiss parameters of the beam particles in the middle of SCM-Y were determined to be $\beta_{u}=4.46 \mathrm{~m}, \alpha_{u}=-0.514, \beta_{w}=$ $4.54 \mathrm{~m}$, and $\alpha_{w}=0.121$ for the above-described matching condition, while the beam emittance was taken to be $\varepsilon_{u}=$ $\varepsilon_{w}=2 \pi \mathrm{mm}$ mrad. Here, the local coordinates $(u, v$, and $w$ ), defined in Fig. 2, is henceforth used in the following calculations. Having used the twiss parameters and emittance, and assumed that the beam particles has a Gaussian distribution in phase space, the phase-space distribution of the initial beam particles in the middle of SCM-Y could be generated. 
With the initial phase-space distribution, we now can calculate the trajectories of each beam particle through the magnetic field by using the following equation of motion:

$$
\begin{gathered}
\frac{d u}{d v}=\frac{\xi_{u}}{\xi_{v}}, \\
\frac{d w}{d v}=\frac{\xi_{w}}{\xi_{v}}, \\
\frac{d(c t)}{d v}=\frac{\gamma}{\xi_{v}}, \\
\frac{d \xi_{u}}{d v}=\frac{Z e c}{m_{0} c^{2} \xi_{v}}\left(\xi_{v} B_{w}-\xi_{w} B_{v}\right), \\
\frac{d \xi_{v}}{d v}=\frac{Z e c}{m_{0} c^{2} \xi_{v}}\left(\xi_{w} B_{u}-\xi_{u} B_{w}\right), \\
\frac{d \xi_{w}}{d v}=\frac{Z e c}{m_{0} c^{2} \xi_{v}}\left(\xi_{u} B_{v}-\xi_{v} B_{u}\right),
\end{gathered}
$$

where $\xi_{i}=\beta_{i} \gamma(i=u, v, w)$. In the simulation code, this equation of motion was numerically integrated using the 4th order Runge-Kutta method.

Three-dimensional maps on the magnetic fields over a region of interest were extracted from the OPERA-3D code, and used in the simulation code. The magnetic-flux density, at which a particle locates, was determined by interpolating those of proximal data, as extracted from a three-dimensional field map. Since the design as well as specifications of BM9 and BM10 are identical, we employed the same field map for the dipole fields of BM9 and $\mathrm{BM} 10$, as described in the preceding section. However, field maps of the quadrupole field for BM9 and BM10 were separately generated, so as to provide design values of a $G L$ product, as given by beam-optics calculations, and added to the dipole field in the simulation code. Concerning the dipole field of BM7 and BM8, we assumed that it is feasible to optimize their conductor positions using the same method, as describe above, and consequently to obtain a similar field quality as those for BM9 and $\mathrm{BM} 10$, because the apertures of BM7 and BM8 are smaller than that for BM9 and BM10. Therefore, we approximated the dipole field for BM9 and BM10 by using the same field map for BM7-BM10 for simplicity.

Under these conditions, a first attempt was made for a simulation using the field map of the optimized dipole field, as provided by a field calculation for a coil current of $I=231.2$ A. To transport a beam particles without any kicks of SCM-X and SCM-Y to the isocenter, $\Delta v=$ $\Delta w=0$, the beam energy of the particles was adjusted instead, to be $E=430.96 \mathrm{MeV} / u$. With the above conditions, the beam-tracking simulations for various kick angles of SCM-X and SCM-Y were performed. A calculated beam profile at the isocenter is presented in Fig. 18.

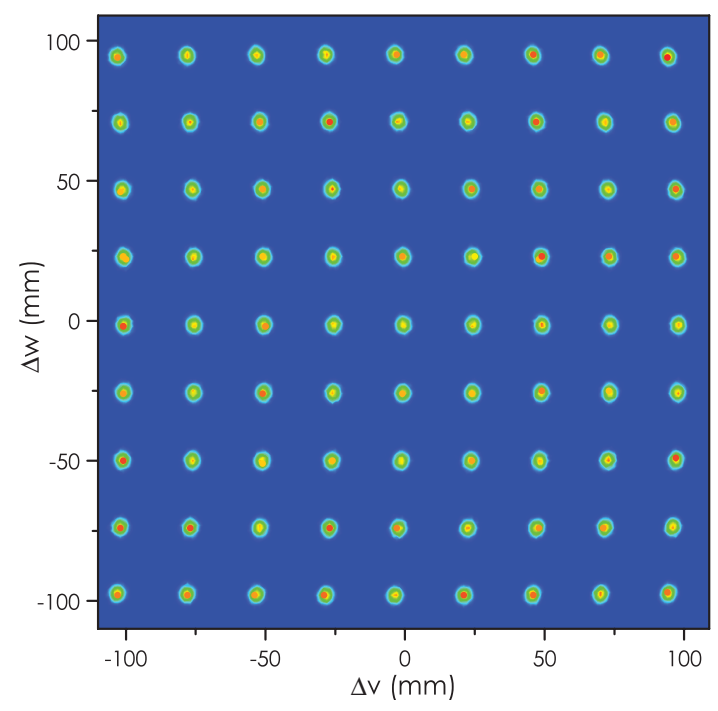

FIG. 18. Beam profile at the isocenter, as calculated with tracking simulations. Each of the beam spots consisted of 5000 particles, and the 81 spots correspond to the beam with the maximum kicks of SCM-X and SCM-Y to be \pm 18 and $\pm 21 \mathrm{mrad}$, respectively, as multiplied by $0,0.25,0.75$, or 1.0 . In the simulations, the field map for the coil currents of $231.2 \mathrm{~A}$ was used, and the corresponding beam energy is $E=430.96 \mathrm{MeV} / u$.

We can see 81 beam spots, which correspond to the beams, as kicked by SCM-X and SCM-Y with kick angles of \pm 18 and $\pm 21 \mathrm{mrad}$, respectively, as multiplied by $0,0.25$, $0.50,0.75$, or 1.0 . For each spot, 5000 particles were tracked from the middle of SCM-Y to the isocenter. With the calculated profile, we found that the size of each beam spot as well as its shape is consistent with the designed values, $\sqrt{\beta \varepsilon}=\sqrt{5 \times 2} \mathrm{~mm}$, as expected by the beam-optics calculations. However, we observed that

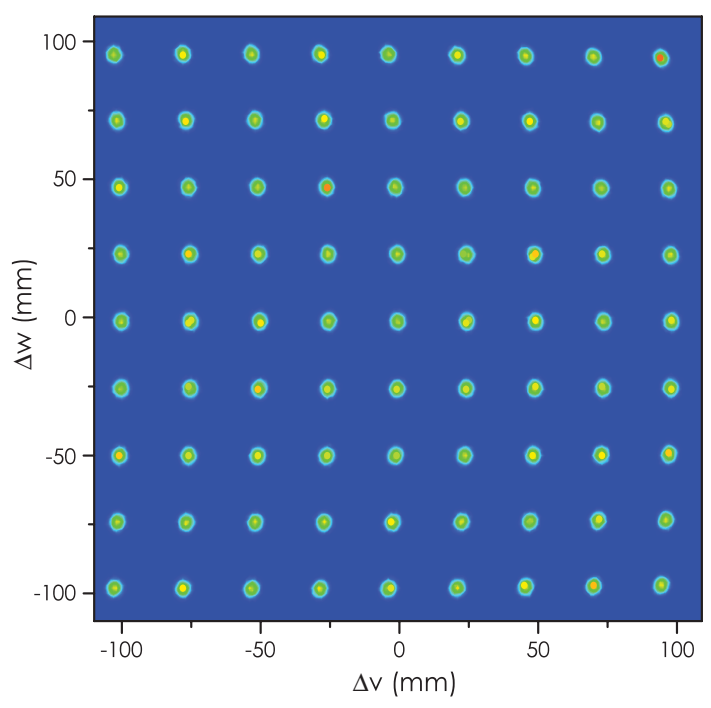

FIG. 19. A similar plot of the beam profile as shown in Fig. 18, but a field map for the coil currents of 91.34 A was used in the tracking simulation. 


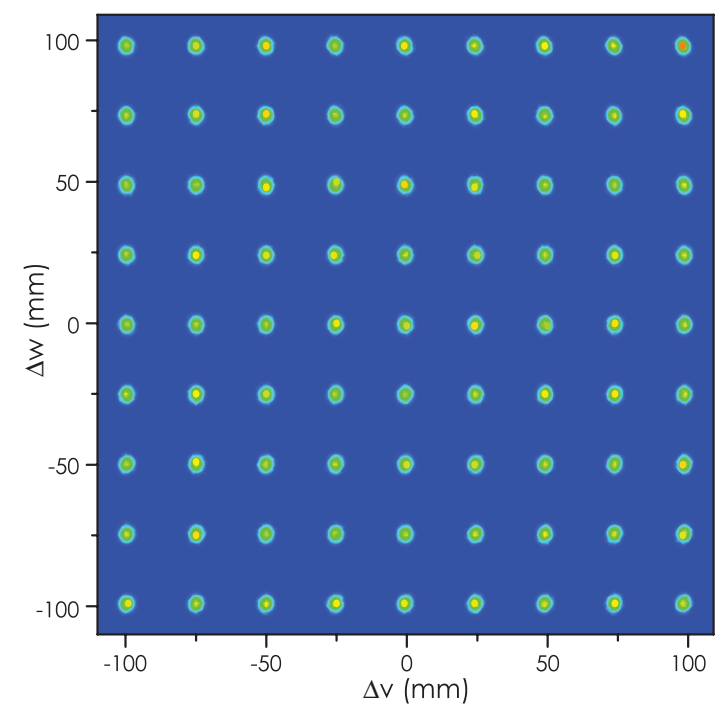

FIG. 20. A similar plot of the beam profile as shown in Fig. 18, but positions of each beam spots were corrected by adjusting kick angles of the scanning magnets.

the positions of the beam spots were slightly shifted from the designed locations; also, the maximum displacement from the designed location was found to be $4.2 \mathrm{~mm}$. This displacement would be attributed to an error in the magnetic field.

A second attempt was made for a similar calculation, but instead using the field map for a coil current of $I=91.34 \mathrm{~A}$; the result is presented in Fig. 19. The beam energy of the particles was taken to be $E=79.61 \mathrm{MeV} / u$. The calculated profile would have a similar tendency, although the maximum displacement was slightly increased to be $4.3 \mathrm{~mm}$.

These displacements can be corrected by adjusting the kick angles of SCM-X and SCM-Y. Having mapped the displacement of each beam spot, and subsequently corrected the kick angles of the scanning magnets, the beam profile, as given in Fig. 20, was obtained. The corresponding coil current of this correction for the scanning magnets was found to be less than $4 \%$. We finally concluded that the positions as well as the size of the beam spot can be under control, and be appropriately reproduced as designed, proving validity of our gantry design.

\section{SUMMARY}

We designed a superconducting rotating gantry for heavy-ion therapy. This isocentric gantry can transport heavy-ion beams with the maximum kinetic energy of $430 \mathrm{MeV} / u$ to an isocenter over 0-360 degrees. Considering the beam optics in the rotating-gantry line, the layout was optimized so as to design a compact gantry, while keeping a large scan size at the isocenter. Consequently, the length and radius of the gantry would become approximately 13 and $5.5 \mathrm{~m}$, respectively, whereas the maximum scan size was calculated to be approximately $200 \mathrm{~mm}$ square.

Combined-function superconducting magnets for the gantry were designed. The positions of the superconducting wires were initially optimized so as to produce pure dipole or quadrupole fields. We subsequently calculated the magnetic-field distributions using a three-dimensional field solver, and found that the distributions have higherorder multipoles, which would be attributed to the curved structure of the magnets as well as the coil ends. Having corrected the conductor positions to compensate for the observed higher-order multipoles, we obtained a uniform field over the effective region.

To evaluate the calculated field quality for the largeaperture magnet, we performed beam-tracking simulations using three-dimensional field maps, as extracted by field calculations. Although the displacements of spot positions were observed, we found that the size of each beam spot as well as its shape is consistent with the designed values, as expected by the beam-optics calculations. Since these displacements can be corrected by adjusting the kick angles of the scanning magnets by approximately $4 \%$, we concluded that the size of the beam spot can be under control, and be appropriately reproduced as designed.

\section{ACKNOWLEDGMENTS}

We thank the other members of the Toshiba Corporation for their help in the design. This work is supported by Ministry of Education, Culture, Sports, Science and Technology (MEXT), Japan.

[1] Y. Hirao, H. Ogawa, S. Yamada, Y. Sato, T. Yamada, T. Murakami, A. Kitagawa, K. Sato, A. Itano, M. Kumada, E. Takada, M. Kanazawa, K. Noda, M. Sudou, K. Kawachi, F. Soga, M. Endo, T. Kanai, S. Minohara, H. Koyama-Ito, and T. Kohno, Annual Report HIMAC, National Institute of Radiological Sciences, NIRS-M-89/HIMAC-001, 1992.

[2] K. Noda, T. Furukawa, Y. Iwata, T. Kanai, M. Kanazawa, N. Kanematsu, A. Kitagawa, M. Komori, T. Murakami, M. Muramatsu, S. Sato, Y. Sato, S. Shibuya, M. Torikoshi, and S. Yamada, Nucl. Instrum. Methods Phys. Res., Sect. A 562, 1038 (2006).

[3] K. Noda, T. Furukawa, T. Fujimoto, T. Inaniwa, Y. Iwata, T. Kanai, M. Kanazawa, S. Minohara, T. Miyoshi, T. Murakami, Y. Sano, S. Sato, E. Takada, Y. Takei, K. Torikai, and M. Torikoshi, Nucl. Instrum. Methods Phys. Res., Sect. B 266, 2182 (2008).

[4] T. Furukawa, T. Inaniwa, S. Sato, Y. Iwata, T. Fujimoto, S. Minohara, K. Noda, and T. Kanai, Nucl. Instrum. Methods Phys. Res., Sect. B 266, 2186 (2008).

[5] T. Furukawa, T. Inaniwa, S. Sato, T. Tomitani, S. Minohara, K. Noda, and T. Kanai, Med. Phys. 34, 1085 (2007).

[6] T. Furukawa, T. Inaniwa, S. Sato, T. Shirai, Y. Takei, E. Takeshita, K. Mizushima, Y. Iwata, T. Himukai, S. Mori, 
S. Fukuda, S. Minohara, E. Takada, T. Murakami, and K. Noda, Med. Phys. 37, 5672 (2010).

[7] E. Pedroni, T. Böhringer, A. Coray, G. Goitein, M. Grossmann, A. Lomax, S. Lin, and M. Jermann, in Proceedings of the 16th International Conference on Cyclotrons and their Applications, East Lansing, MI (AIP, New York, 2001), p. 13.

[8] F. T. Cole, P. V. Livdahl, F. E. Mills, and L. C. Teng, in Proceedings of the 1989 Particle Accelerator Conference, Chicago, IL (IEEE, New York, 1989), p. 737.

[9] H. Eickhoff et al., in Proceedings of the 8th European Particle Accelerator Conference, Paris, 2002 (EPS-IGA and CERN, Geneva, 2002), p. 2730.

[10] T. Furukawa and K. Noda, Nucl. Instrum. Methods Phys.
Res., Sect. A 565, 430 (2006).

[11] T. Obana, Ph.D. dissertation, The Graduate University for Advanced Studies, School of High Energy Accelerator Science, 2005.

[12] T. Obana, T. Ogitsu, T. Nakamoto, K. Sasaki, A. Yamamoto, T. Orikasa, M. Yoshimoto, and Y. Mori, IEEE Trans. Appl. Supercond. 15, 1185 (2005).

[13] Y. Iwata, T. Kadowaki, H. Uchiyama, T. Fujimoto, E. Takada, T. Shirai, T. Furukawa, K. Mizushima, E. Takeshita, K. Katagiri, S. Sato, Y. Sano, and K. Noda, Nucl. Instrum. Methods Phys. Res., Sect. A 624, 33 (2010).

[14] Opera Version 15, http://www.cobham.com/. 